\title{
UNIQUENESS OF SOLUTIONS FOR SOME ELLIPTIC EQUATIONS WITH A QUADRATIC GRADIENT TERM*
}

\author{
David Arcoya ${ }^{1}$ And Sergio Segura de LeÓN ${ }^{2}$
}

\begin{abstract}
We study a comparison principle and uniqueness of positive solutions for the homogeneous Dirichlet boundary value problem associated to quasi-linear elliptic equations with lower order terms. A model example is given by

$$
-\Delta u+\lambda \frac{|\nabla u|^{2}}{u^{r}}=f(x), \quad \lambda, r>0 .
$$

The main feature of these equations consists in having a quadratic gradient term in which singularities are allowed. The arguments employed here also work to deal with equations having lack of ellipticity or some dependence on $u$ in the right hand side. Furthermore, they could be applied to obtain uniqueness results for nonlinear equations having the p-Laplacian operator as the principal part. Our results improve those already known, even if the gradient term is not singular.
\end{abstract}

Mathematics Subject Classification. 35J65, 35J70, 35J60.

Received May 27, 2008. Revised October 2nd, 2008.

Published online December 19, 2008.

Dedicato a Lucio Boccardo in occasione del suo $60^{\circ}$ compleanno

\section{INTRODUCTION}

In this paper, we consider the following Dirichlet problem

$$
\left\{\begin{aligned}
-\operatorname{div}(\alpha(u) \nabla u)+\beta(u)|\nabla u|^{2} & =f(x, u) & & \text { in } \Omega ; \\
u & =0 & & \text { on } \partial \Omega .
\end{aligned}\right.
$$

Here the real continuous functions $\alpha$ and $\beta$ are defined in an open interval $I=] 0, b[$ (the value $b=+\infty$ is not excluded), while the function $f: \Omega \times I \rightarrow \mathbb{R}$ satisfies the Carathéodory condition. We also assume that $\beta(s) \geq 0$ and $\alpha(s)>0$ for every $s \in I$. We point out that $\alpha$ and $\beta$ may be singular in the extremes of the interval.

\footnotetext{
Keywords and phrases. Non linear elliptic problems, uniqueness, comparison principle, lower order terms with singularities at the Gradient term, lack of coerciveness.

* First author is supported by D.G.E.S. Ministerio de Educación y Ciencia (Spain) MTM2006-09282 and Junta de Andalucía FQM116. Second author is supported by Ministerio de Ciencia e Innovación (Spain) MTM2008-03176.

${ }^{1}$ Departamento de Análisis Matemático, Universidad de Granada, Campus Fuentenueva s/n, 18071 Granada, Spain. darcoya@ugr.es

2 Departament d'Anàlisi Matemàtica, Universitat de València, Dr. Moliner 50, 46100 Burjassot, Valencia, Spain. sergio.segura@uv.es
} 
Our aim is to prove a comparison principle for solutions of this quasilinear problem and, as a consequence, uniqueness of solutions such that $u(x) \in I$ for almost every $x \in \Omega$. (For the precise meaning of our concept of solution, see Def. 2.1 below.)

As a model problem with singular terms we consider for $\lambda, r>0$ the boundary value problem

$$
\left\{\begin{aligned}
-\Delta u+\lambda \frac{|\nabla u|^{2}}{u^{r}} & =f(x), & & \text { in } x \in \Omega, \\
u & =0, & & \text { on } x \in \partial \Omega .
\end{aligned}\right.
$$

The study of this kind of singular problems is very recent. Indeed, the existence of solution is proved in $[3,4,6]$ for the case that $r \leq 1$ and $f(x) \in L^{q}(\Omega)$, with $q>N / 2$, and

$$
\inf _{\omega} f>0, \quad \forall \omega \subset \subset \Omega
$$

Recently, in [15] this result is improved by replacing condition (1.3) by the weaker condition that $0 \leq f \in$ $L^{\left(2^{*} / r\right)^{\prime}}(\Omega)$ with $f \not \equiv 0$ and $\lambda<1 / 2$ in the case $r=1$. On the other hand, a similar equation is studied in [24]. The case $r>1$ is studied by the first time in [5] where, among other more general results, existence of solutions is proved for every $f \in L^{\left(2^{*}\right)^{\prime}}(\Omega)$ satisfying (1.3) provided that $r<2$, while for $r \geq 2$, it is shown that in general there is nonexistence of solutions.

However, at least for our knowledge, the study of the uniqueness of solutions for (1.2) is completely new. Even in the case of nonsingular terms, i.e. if $\alpha$ and $\beta$ are continuous in $[0,+\infty[$, there are only few results concerning the uniqueness. Indeed, in [7] (see also [9] for some extended results allowing $L^{N}$-dependence, instead of $L^{\infty}$-one, in $x$ ), it is proved a comparison principle for solutions of (1.1) whose simplest version requires $\alpha=1$ and $\beta$ a $C^{1}$-function satisfying

$$
\beta(0)=0, \quad \beta^{\prime}(s)>0, \quad \forall s>0 .
$$

A simple comparison principle can also be found in the proof of Theorem 3.1 of [28] (see also [27]) for the case $\alpha \equiv 1$ and $\beta$ nondecreasing.

Other uniqueness results for equations with a gradient term can be found in [31], where the assumption $\beta(s) \leq 0$ is considered, [26] for uniqueness of the zero solution for a sign-changing nonsingular function $\beta$ and $f(x, s) s \leq 0$, [8] for the case of subquadratic terms in $\nabla u,[11-13]$ for equations with different dependence on $\nabla u$ and $[14,22,29]$ for quasilinear equations with no quadratic term in $\nabla u$.

Here we will proved uniqueness of solutions $u \in H_{0}^{1}(\Omega)$ for (1.2) provided either that $r<1$ or that $r=1$ and $\lambda<1$. We prove this result (see Cor. 2.12) as a consequence of a general uniqueness theorem, Theorem 2.9, which is deduced from a comparison principle (see Thm. 2.7) for the general problem (1.1). We point out that Theorem 2.9 handles also the case of nonsingular terms and, in this case, we improve the result of [7] since we do not require assumption (1.4). See Corollary 2.10 and Remark 2.8 for more details.

Furthermore, adapting ideas of [21], we study the uniqueness of solutions in the class of bounded solutions, i.e. in $H_{0}^{1}(\Omega) \cap L^{\infty}(\Omega)$. In this case, we improve the results of [7,9] (see Thm. 3.1 and Rem. 3.2-2).

\section{Comparison PRINCIPle AND UNiqueness Result}

We begin by introducing some notation which will be used throughout this paper. For each $k>0$, we define the truncation at levels $\pm k$ by

$$
T_{k}(s)=(\operatorname{sign} s)(k \wedge|s|), \quad s \in \mathbb{R} .
$$

To prove our results, we fix a point $a \in I$, and define an auxiliary function $\gamma: I \longrightarrow \mathbb{R}$ as

$$
\gamma(s)=\int_{a}^{s} \frac{\beta(t)}{\alpha(t)} \mathrm{d} t, \quad s \in I .
$$

We state what we understand by sub- and supersolution. 
Definition 2.1. By a subsolution (respectively, supersolution) of problem (1.1) we mean a measurable function $u: \Omega \rightarrow \mathbb{R}$ such that $u(x) \in I$ for almost every $x \in \Omega, T_{k}(u) \in H_{0}^{1}(\Omega)$ for $0<k<b$ and the following conditions hold:

$$
\begin{gathered}
\alpha(u)|\nabla u| \in L^{2}(\Omega), \quad \beta(u)|\nabla u|^{2} \in L^{1}(\Omega), \quad f(\cdot, u) \in L^{1}(\Omega), \\
\int_{\Omega} \alpha(u) \nabla u \cdot \nabla v+\int_{\Omega} \beta(u)|\nabla u|^{2} v \stackrel{(\geq)}{\leq} \int_{\Omega} f(x, u) v,
\end{gathered}
$$

for every $v \in H_{0}^{1}(\Omega) \cap L^{\infty}(\Omega)$ with $v \geq 0$. A solution is a function which is both a subsolution and a supersolution for $(1.1)$.

Remarks 2.2. (1) We point out that the condition $T_{k}(u) \in H_{0}^{1}(\Omega)$ for every $k \in I$ implies that a gradient $\nabla u$ can be defined (see [10]): It is the only measurable function $v: \Omega \rightarrow \mathbb{R}^{N}$ satisfying

$$
\nabla T_{k}(u)=v \chi_{\{|u|<k\}}, \quad \forall k \in I
$$

This hypothesis, that the truncations of the solution are in the "energy space" $H_{0}^{1}(\Omega)$, is quite natural when dealing with elliptic problems having a non regular datum (see $[10,23]$ ) or either a noncoercive principal term (see $[2,19,20,30])$. In our setting truncations are also used in a essential way in order to prove our main tool (see Prop. 2.3 below).

(2) Observe that every term in (2.3) is well-defined because of condition (2.2). It is worthwhile to point out that it is natural to require some additional integrability to $f$ in order to assure the existence of solution $u$ such that $\alpha(u)|\nabla u| \in L^{2}(\Omega)$. For instance, in the case that $\alpha \equiv 1, \beta \equiv 0$ and $f(x, u)=f(x)$, we have to impose that $f \in L^{m}(\Omega)$ with $m \geq 2 N /(N+2)$ to guarantee the existence of solutions $u$ belonging to the space $H_{0}^{1}(\Omega)$ (i.e., such that $\alpha(u)|\nabla u| \in L^{2}(\Omega)$ ) of the homogeneous Dirichlet boundary value problem associated to the equation $-\Delta u=f$. Remind that, in general this is not so for the case $f \in L^{m}(\Omega)$ with $1<m<2 N /(N+2)$.

The following result will be used several times in the sequel. It states an inequality where the quadratic term on the gradient is canceled. (Similar cancellation results can be found in [20,31].) Formally, the idea of its proof is to take, for $w \in H_{0}^{1}(\Omega) \cap L^{\infty}(\Omega), v=\mathrm{e}^{-\gamma(u)} w$ as test function in (2.3). However, observe that, since the function $t \mapsto \mathrm{e}^{-\gamma(t)}$ may be unbounded, this choice of test function could be impossible. Thus, we are forced to take a suitable truncature of it.

Proposition 2.3. Let $u$ be a subsolution (respectively a supersolution) of (1.1) for which there exists $\delta \in] 0, b[$ such that

Then the inequality

$$
\begin{gathered}
\alpha\left(T_{\delta}(u)\right) \mathrm{e}^{-\gamma\left(T_{\delta}(u)\right)}\left|\nabla T_{\delta}(u)\right| \in L^{2}(\Omega) \\
\beta\left(T_{\delta}(u)\right)\left|\nabla T_{\delta}(u)\right|^{2} \mathrm{e}^{-\gamma\left(T_{\delta}(u)\right)}, f\left(x, T_{\delta}(u)\right) \mathrm{e}^{-\gamma\left(T_{\delta}(u)\right)} \in L^{1}(\Omega) .
\end{gathered}
$$

$$
\int_{\Omega} \alpha(u) \mathrm{e}^{-\gamma(u)} \nabla u \cdot \nabla w \stackrel{(\geq)}{\leq} \int_{\Omega} f(x, u) \mathrm{e}^{-\gamma(u)} w
$$

holds for every $w \in H_{0}^{1}(\Omega) \cap L^{\infty}(\Omega)$, with $w \geq 0$.

Remarks 2.4. (1) We point out that assumption (2.4) in the above proposition is straightforwardly satisfied if the function $\beta / \alpha$ is integrable in a right neighborhood of 0 . Indeed, under this assumption (2.4), the function $\gamma$ is bounded in $] 0, a$. Consequently, taking into account that $\gamma \geq 0$ in $[a, b[$, we deduce that $\mathrm{e}^{-\gamma(u)}$ is bounded and by using the integrability of $\alpha(u)^{2}|\nabla u|^{2}, \beta(u)|\nabla u|$ and $f(x, u)$ (remind that $u$ is a subsolution of (1.1)), we see that (2.4) is trivially satisfied.

(2) Even more, if $\beta / \alpha \in L^{1}(I) \cap L^{\infty}(I)$, then $\mathrm{e}^{-\gamma(u)} \in H^{1}(\Omega) \cap L^{\infty}(\Omega)$, and so the proof below can be simplified by taking directly $v=\mathrm{e}^{-\gamma(u)} w$ as test function in (2.3). 
Proof. We prove only the assertion of the theorem for a subsolution $u$ and leave the corresponding one for a supersolution to the reader. Let $w \in H_{0}^{1}(\Omega) \cap L^{\infty}(\Omega)$ be fixed. For every $\left.\epsilon \in\right] 0, \min \{b-a, \delta\}[$, we denote

$$
b_{\epsilon}= \begin{cases}b-\epsilon, & \text { if } b<+\infty, \\ a+\frac{1}{\epsilon}, & \text { if } b=+\infty,\end{cases}
$$

and

$$
\tau_{\epsilon}(s)=(s \vee \epsilon) \wedge b_{\epsilon}, \quad \forall s>0 .
$$

Note that $a<b_{\epsilon}$ and thus $\gamma\left(b_{\epsilon}\right) \geq 0$. Since $\tau_{\epsilon}(u) \geq \epsilon$ and $\gamma$ is increasing, we have $\mathrm{e}^{-\gamma\left(\tau_{\epsilon}(u)\right)} \leq \mathrm{e}^{-\gamma(\epsilon)}$. On the other hand, by the positivity of $\alpha$, we also have $\frac{\beta\left(\tau_{\epsilon}(u)\right)}{\alpha\left(\tau_{\epsilon}(u)\right)} \leq \frac{\max _{t \in\left[\epsilon, b_{\epsilon}\right]} \beta(t)}{\min _{t \in\left[\epsilon, b_{\epsilon}\right]} \alpha(t)}$, and then $\frac{\beta\left(\tau_{\epsilon}(u)\right)}{\alpha\left(\tau_{\epsilon}(u)\right)} \mathrm{e}^{-\gamma\left(\tau_{\epsilon}(u)\right)}\left|\nabla \tau_{\epsilon}(u)\right|$ belongs to $L^{2}(\Omega)$. It follows that

$$
\mathrm{e}^{-\gamma\left(\tau_{\epsilon}(u)\right)} w \in H_{0}^{1}(\Omega) \cap L^{\infty}(\Omega)
$$

Thus, we may choose $v=\mathrm{e}^{-\gamma\left(\tau_{\epsilon}(u)\right)} w$ as test function in (2.3) to get

$$
\begin{aligned}
\int_{\Omega} \alpha(u) \mathrm{e}^{-\gamma\left(\tau_{\epsilon}(u)\right)} \nabla u \cdot \nabla w-\int_{\Omega} \alpha(u) \frac{\beta\left(\tau_{\epsilon}(u)\right)}{\alpha\left(\tau_{\epsilon}(u)\right)}\left[\mathrm{e}^{-\gamma\left(\tau_{\epsilon}(u)\right)} w\right]\left|\nabla \tau_{\epsilon}(u)\right|^{2} & \\
& +\int_{\Omega} \beta(u)|\nabla u|^{2} \mathrm{e}^{-\gamma\left(\tau_{\epsilon}(u)\right)} w \leq \int_{\Omega} f(x, u) \mathrm{e}^{-\gamma\left(\tau_{\epsilon}(u)\right)} w,
\end{aligned}
$$

for every $\epsilon \in] 0, \min \{b-a, \delta\}\left[\right.$. In order to take the limit as $\epsilon$ goes to 0 , we point out that $\mathrm{e}^{-\gamma\left(b_{\epsilon}\right)} \leq 1$ and

$$
\begin{gathered}
-\int_{\Omega} \alpha(u) \frac{\beta\left(\tau_{\epsilon}(u)\right)}{\alpha\left(\tau_{\epsilon}(u)\right)}\left[\mathrm{e}^{-\gamma\left(\tau_{\epsilon}(u)\right)} w\right]\left|\nabla \tau_{\epsilon}(u)\right|^{2}+\int_{\Omega} \beta(u)|\nabla u|^{2} \mathrm{e}^{-\gamma\left(\tau_{\epsilon}(u)\right)} w= \\
\quad \int_{\{u<\epsilon\}} \beta(u)|\nabla u|^{2} \mathrm{e}^{-\gamma(\epsilon)} w+\int_{\left\{u>b_{\epsilon}\right\}} \beta(u)|\nabla u|^{2} \mathrm{e}^{-\gamma\left(b_{\epsilon}\right)} w \\
=\int_{\{u<\epsilon\}} \beta\left(T_{\delta}(u)\right)\left|\nabla T_{\delta}(u)\right|^{2} \mathrm{e}^{-\gamma(\epsilon)} w+\int_{\left\{u>b_{\epsilon}\right\}} \beta(u)|\nabla u|^{2} \mathrm{e}^{-\gamma\left(b_{\epsilon}\right)} w \\
\leq\|w\|_{\infty} \int_{\{u<\epsilon\}} \beta\left(T_{\delta}(u)\right)\left|\nabla T_{\delta}(u)\right|^{2} \mathrm{e}^{-\gamma\left(T_{\delta}(u)\right)}+\|w\|_{\infty} \int_{\left\{u>b_{\epsilon}\right\}} \beta(u)|\nabla u|^{2} .
\end{gathered}
$$

Using (2.4), we have $\beta\left(T_{\delta}(u)\right)\left|\nabla T_{\delta}(u)\right|^{2} \mathrm{e}^{-\gamma\left(T_{\delta}(u)\right)} \in L^{1}(\Omega)$ and thus

$$
\lim _{\epsilon \rightarrow 0} \int_{\{u<\epsilon\}} \beta\left(T_{\delta}(u)\right)\left|\nabla T_{\delta}(u)\right|^{2} \mathrm{e}^{-\gamma\left(T_{\delta}(u)\right)}=0 .
$$

On the other hand, by the definition of subsolution of (1.1), $\beta(u)|\nabla u|^{2} \in L^{1}(\Omega)$ and then

$$
\lim _{\epsilon \rightarrow 0} \int_{\left\{u>b_{\epsilon}\right\}} \beta(u)|\nabla u|^{2}=0 .
$$


Therefore, by (2.7), we deduce that

$$
\int_{\Omega} \alpha(u) \mathrm{e}^{-\gamma\left(\tau_{\epsilon}(u)\right)} \nabla u \cdot \nabla w \leq \int_{\Omega} f(x, u) \mathrm{e}^{-\gamma\left(\tau_{\epsilon}(u)\right)} w+\omega(\epsilon),
$$

where $\lim _{\epsilon \rightarrow 0} \omega(\epsilon)=0$. Using now that $\mathrm{e}^{-\gamma(s)} \leq \mathrm{e}^{-\gamma(\delta)}$ for every $\left.s \in\right] \delta, b[$, we obtain

$$
\begin{aligned}
\alpha(u) \mathrm{e}^{-\gamma(u)}|\nabla u| & =\alpha(u) \mathrm{e}^{-\gamma(u)}|\nabla u| \chi_{\{u<\delta\}}+\alpha(u) \mathrm{e}^{-\gamma(u)}|\nabla u| \chi_{\{u \geq \delta\}} \\
& \leq \alpha\left(T_{\delta}(u)\right) \mathrm{e}^{-\gamma\left(T_{\delta}(u)\right)}\left|\nabla T_{\delta}(u)\right|+\alpha(u) \mathrm{e}^{-\gamma(\delta)}|\nabla u|
\end{aligned}
$$

and we deduce from (2.4) and the definition of subsolution that $\alpha(u) \mathrm{e}^{-\gamma(u)}|\nabla u| \in L^{2}(\Omega)$. Similarly, $f(x, u) \mathrm{e}^{-\gamma(u)} \in L^{1}(\Omega)$. As a consequence, we may let $\epsilon$ tend to 0 in (2.8) by applying Lebesgue's theorem and we conclude that

as desired.

$$
\int_{\Omega} \alpha(u) \mathrm{e}^{-\gamma(u)} \nabla u \cdot \nabla w \leq \int_{\Omega} f(x, u) \mathrm{e}^{-\gamma(u)} w
$$

In the following results, we make two additional assumptions:

(H1) The function $t \mapsto \alpha(t) \mathrm{e}^{-\gamma(t)}$ is integrable in a right neighborhood of zero.

(H2) The function $s \mapsto f(x, s) \mathrm{e}^{-\gamma(s)}$ is decreasing for a.e. $x \in \Omega$.

Remark 2.5. Assumption (H1) on the function $\alpha(s) \mathrm{e}^{-\gamma(s)}$ prevents large singularities at 0 . To illustrate this fact, suppose that $\alpha(s)=1$ and $\beta(s)=\lambda / s^{r}$, with $\lambda, r>0$. Then, if $r \neq 1$, we have $\alpha(s) \mathrm{e}^{-\gamma(s)}=$ $\mathrm{e}^{-\frac{\lambda}{1-r}\left(s^{1-r}-a^{1-r}\right)}$, while, for the case $r=1, \alpha(s) \mathrm{e}^{-\gamma(s)}=a^{r} / s^{\lambda}$. Consequently, the function becomes integrable on every interval containing 0 if and only if either $r<1$ or $\lambda<1$ and $r=1$.

Remark 2.6. If we assume that the function $f(x, s)$ is continuously differentiable with respect to the second variable, condition (H2) becomes

$$
\frac{\partial f}{\partial s}(x, s) \leq \frac{\beta(s)}{\alpha(s)} f(x, s)
$$

for every $s \in I$ and a.e. $x \in \Omega$.

In particular, if $f$ is nonnegative and does not depends on $s \in I$, i.e. if $f(x, s)=f(x) \geq 0$, then (H2) is always satisfied.

On the other hand, assuming that $f(x, s)=\lambda h(x) g(s)$, with $h$ positive and $\lambda>0$, a sufficient condition for (2.10) is $g^{\prime}(s) \leq \frac{\beta(s)}{\alpha(s)} g(s)$ for all $s \in I$. Some particular cases of functions $g$ explain this. First, we consider $g(s)=(1+s)^{\theta}$, with $\theta>0$. The sufficient condition becomes $\frac{\theta}{1+s} \leq \frac{\beta(s)}{\alpha(s)}$. On the other hand, if $g(s)=\log (1+s)$, then it turns into $\frac{1}{(1+s) \log (1+s)} \leq \frac{\beta(s)}{\alpha(s)}$, while, if $g(s)=\log (1+\log (1+s))$, then it becomes $\frac{1}{(1+s)(1+\log (1+s)) \log (1+\log (1+s))} \leq \frac{\beta(s)}{\alpha(s)}$. In this way, we see that there is some connection between how singular can be $\frac{\beta}{\alpha}$ and the function $g$.

Now, we state our comparison principle.

Theorem 2.7. Assume that $(\mathrm{H} 1)$ and $(\mathrm{H} 2)$ hold. Let $u, \widetilde{u} \in H^{1}(\Omega)$ be respectively a subsolution and a supersolution of problem (1.1) satisfying (2.4). If $u \leq \widetilde{u}$ on $\partial \Omega$ (in the sense that $(u-\widetilde{u})^{+} \in H_{0}^{1}(\Omega)$ ), then $u \leq \widetilde{u}$. 
Remark 2.8. Observe that if the functions $\alpha$ and $\beta$ are continuous in $[0,+\infty[($ i.e., $b=+\infty$, and moreover $\alpha(0)$ and $\beta(0)$ are defined) and $\alpha(0)>0$, then $\beta / \alpha$ is integrable in a neighborhood of $s=0$ and, by Remark 2.4-(1), we deduce that (2.4) holds for both a subsolution $u$ and a supersolution $\widetilde{u}$ of (1.1). Consequently, in this case, the above comparison principle improves the one given in [7], Theorem 2.6, where the authors impose, in addition, that ( $\alpha=1$ and that) there exist a positive constant $n$ and a continuous function $z$ such that $\exp \left[-n^{-1} \int_{0}^{t} z\right] \in L^{\infty}(0,+\infty) \cap L^{1}(0,+\infty)$ with

$$
\beta^{\prime}(s)-\frac{1}{2 n}[2 \beta(s)-z(s)]^{2}>0, \quad \forall s>0 .
$$

Proof. We use an auxiliary function defined thanks to (H1) by

$$
\Psi(s)=\int_{0}^{s} \alpha(t) \mathrm{e}^{-\gamma(t)} \mathrm{d} t, \quad \forall s \in I .
$$

Observe that if $u$ is a subsolution of (1.1) satisfying (2.4), then, due to the $L^{2}$-integrability of the function $\alpha(u) \mathrm{e}^{-\gamma(u)}|\nabla u|$ (remind (2.9)), we derive that $\Psi(u) \in H^{1}(\Omega)$. An analogous argument also gives $\Psi(\widetilde{u}) \in H^{1}(\Omega)$ for a supersolution of (1.1) satisfying (2.4). Since $u \leq \widetilde{u}$ on $\partial \Omega$ and using that $\Psi$ is increasing, we have $[\Psi(u)-\Psi(\widetilde{u})]^{+} \in H_{0}^{1}(\Omega)$ and we deduce that

$$
T_{k}[\Psi(u)-\Psi(\widetilde{u})]^{+} \in H_{0}^{1}(\Omega) \cap L^{\infty}(\Omega), \quad \forall k>0 .
$$

Applying Proposition 2.3, it yields

$$
\int_{\Omega} \alpha(u) \mathrm{e}^{-\gamma(u)} \nabla u \cdot \nabla T_{k}[\Psi(u)-\Psi(\widetilde{u})]^{+} \leq \int_{\Omega} f(x, u) \mathrm{e}^{-\gamma(u)} T_{k}[\Psi(u)-\Psi(\widetilde{u})]^{+} .
$$

Having in mind $\nabla \Psi(u)=\Psi^{\prime}(u) \nabla u=\alpha(u) \mathrm{e}^{-\gamma(u)} \nabla u$ we obtain

$$
\int_{\Omega} \nabla \Psi(u) \cdot \nabla T_{k}[\Psi(u)-\Psi(\widetilde{u})]^{+} \leq \int_{\Omega} f(x, u) \mathrm{e}^{-\gamma(u)} T_{k}[\Psi(u)-\Psi(\widetilde{u})]^{+} .
$$

Similarly, we get

$$
\int_{\Omega} \nabla \Psi(\widetilde{u}) \cdot \nabla T_{k}[\Psi(u)-\Psi(\widetilde{u})]^{+} \geq \int_{\Omega} f(x, \widetilde{u}) \mathrm{e}^{-\gamma(\widetilde{u})} T_{k}[\Psi(u)-\Psi(\widetilde{u})]^{+} .
$$

Subtracting the previous inequalities, we have

$$
\int_{\Omega}\left|\nabla T_{k}[\Psi(u)-\Psi(\widetilde{u})]^{+}\right|^{2} \leq \int_{\Omega}\left(f(x, u) \mathrm{e}^{-\gamma(u)}-f(x, \widetilde{u}) \mathrm{e}^{-\gamma(\widetilde{u})}\right) T_{k}[\Psi(u)-\Psi(\widetilde{u})]^{+} .
$$

On account that $\Psi$ is (strictly) increasing and that $s \mapsto f(x, s) \mathrm{e}^{-\gamma(s)}$ is decreasing, we deduce that the integrand in the right hand side of the above equality is nonpositive. Consequently, we obtain $\int_{\Omega} \mid \nabla T_{k}[\Psi(u)-$ $\Psi(\widetilde{u})]\left.^{+}\right|^{2}=0$ and so, by Poincaré's inequality, $T_{k}[\Psi(u)-\Psi(\widetilde{u})]^{+}=0$ for all $k>0$. Hence, $\Psi(u) \leq \Psi(\widetilde{u})$ a.e. in $\Omega$ and, using again that the function $\Psi$ is strictly increasing, one deduces that $u \leq \widetilde{u}$ a.e. in $\Omega$.

As a straightforward consequence of the above result, we obtain the desired uniqueness result. 
Theorem 2.9. If we assume that $(\mathrm{H} 1)$ and $(\mathrm{H} 2)$ hold, then problem (1.1) has at most a solution $u$ satisfying (2.4).

By Remark 2.8 we deduce the following improvement of [7].

Corollary 2.10. Assume that $f(x, s)=f(x) \geq 0$, that $\alpha$ and $\beta$ are continuous in the interval $[0,+\infty[$ and that $\alpha(0)>0$. Then problem (1.1) has at most a solution $u$.

Remark 2.11. Existence results of bounded and unbounded solutions to elliptic equations with a quadratic gradient term without singularities have extensively been obtained since the eighties of the last century when several articles were published by Boccardo et al. (see, for instance, $[17,18]$ ). On the other hand, (nonsingular) elliptic equations having a principal term with degenerate coercivity have been studied in the nineties $($ see $[2,19]$ and references therein). In some sense, these two features are combined in [16], where the authors look for minima of noncoercive functionals whose corresponding Euler-Lagrange equations have both a noncoercive principal term and a quadratic gradient term. The more general (nonvariational) equation in (1.1) is handled in [20] under the assumptions $\beta / \alpha \in L^{1}(\mathbb{R})$ and $\alpha \notin L^{1}(-\infty, 0) \cup L^{1}(0,+\infty)$. In that paper the following existence results are proved: If $m>\frac{N}{2}$, then there exists a bounded weak solution (Thm. 2.4 in [20]) and if $\frac{N}{2}>m \geq \frac{2 N}{N+2}$, then there exists a weak solution $u$ satisfying $\alpha(u)|\nabla u| \in L^{2}(\Omega)$ (Thm. 3.2 and Prop. 3.7 in [20]). These existence results are improved in [30] where the authors consider, in addition, more general operators, of p-Laplacian type, and the general case $f \in L^{m}(\Omega)$, with $m>1$. We remark that if $f \in L^{m}(\Omega)$, with $1<m<\frac{2 N}{N+2}$, then it is shown in [30] that the function $\alpha(u)|\nabla u|$ no longer stays in $L^{2}(\Omega)$, but it lies in $L^{m^{*}}(\Omega)\left(\right.$ where $\left.m^{*}=\frac{N m}{N-m}\right)$.

By Remark 2.5, we get also uniqueness of solutions for the singular model problem.

Corollary 2.12. If $f(x) \geq 0$ and $\lambda, r>0$, then problem (1.2) has at most one solution satisfying (2.4) provided either that $r<1$ or that $r=1$ and $\lambda<1$.

\section{Another uniqueness Result}

We study in this section the uniqueness of bounded solutions of (1.1), i.e. of solutions $u \in H_{0}^{1}(\Omega) \cap L^{\infty}(\Omega)$. We take advantage of the restriction of the class of functions where we look for uniqueness to improve the condition (H2) and we assume that $\Omega$ is of class $C^{1}$. The result follows the arguments by Brézis and Oswald in [21]. In the sequel, the function $\Psi$ is that defined by (2.11).

Theorem 3.1. Let $I=] 0,+\infty[$ and assume $(\mathrm{H} 1)$ with $\beta / \alpha$ integrable in a right neighborhood of zero, that $\Omega$ is of class $C^{1}$ and that the following conditions hold:

(H3) The function $s \mapsto \frac{f(x, s) \mathrm{e}^{-\gamma(s)}}{\Psi(s)}$ is decreasing for almost all $x \in \Omega$.

(H4) For every $s \in I$, the function $f(x, s)$ is bounded from below and there exists a continuous increasing function $g$ such that $f(x, s) \leq g(s)$, for a.e. $x \in \Omega$ and every $s \in I$.

Then there exists at most a bounded solution u of problem (1.1) satisfying (2.4).

Remarks 3.2. (1) Since the statement of Theorem 3.1 refers to uniqueness of bounded solutions, there is no need of using truncations at level $\delta$ in (2.4). Indeed, if $u$ is a bounded function, then condition (2.4) is equivalent to impose that $\alpha(u) \mathrm{e}^{-\gamma(u)}|\nabla u| \in L^{2}(\Omega)$ and $\beta(u)|\nabla u|^{2} \mathrm{e}^{-\gamma(u)}, f(x, u) \mathrm{e}^{-\gamma(u)} \in L^{1}(\Omega)$.

(2) As in Remark 2.8, if the functions $\alpha$ and $\beta$ are continuous on $[0,+\infty[$ and $\alpha(0)>0$, then (2.4) holds for every solution of (1.1). Thus, Theorem 3.1 improves Theorem 2.2 in [9] (an extension of Thm. 2.3 in [7]) where the authors study the case $\alpha \equiv 1$ and a general quadratic term $H(x, u, \nabla u)$ instead of $\beta(u)|\nabla u|^{2}$. In particular, for the case $H(x, u, \nabla u)=\beta(u)|\nabla u|^{2}$, they additionally impose either that $\beta$ is bounded or that there exist $k \in \mathbb{R}$ and $m>0$ such that

$$
k[\beta(s)-k]+\beta^{\prime}(s) \geq m[\beta(s)-k]^{2}, \quad \forall s>0 .
$$


Proof. Let us consider two bounded solutions $u$ and $v$ of problem (1.1) satisfying the assumptions of the theorem. We point out that $\Psi(u), \Psi(v) \in H_{0}^{1}(\Omega) \cap L^{\infty}(\Omega)$ (see the proof of Thm. 2.7) and that $\Psi(u), \Psi(v)>0$ in $\Omega$. Thus, using (2.5) for the solution $v$ with $w=\Psi(v)$, we obtain

$$
\int_{\Omega} \alpha(v) \mathrm{e}^{-\gamma(v)} \nabla v \cdot \nabla \Psi(v)=\int_{\Omega} \mathrm{e}^{-\gamma(v)} f(x, v) \Psi(v) .
$$

Performing easy manipulations, this equality becomes

$$
\int_{\Omega}|\nabla \Psi(v)|^{2}=\int_{\Omega} \frac{\mathrm{e}^{-\gamma(v)} f(x, v)}{\Psi(v)} \Psi(v)^{2} .
$$

On the other hand, applying Proposition 2.3 with $w \in C_{0}^{\infty}(\Omega)$ and $w \geq 0$, we deduce that

$$
\int_{\Omega} \alpha(u) \mathrm{e}^{-\gamma(u)} \nabla u \cdot \nabla w=\int_{\Omega} f(x, u) \mathrm{e}^{-\gamma(u)} w,
$$

i.e.,

$$
-\Delta \Psi(u)=f(x, u) \mathrm{e}^{-\gamma(u)}, \quad x \in \Omega .
$$

Observe that by the monotonicity of $\frac{f(x, s) \mathrm{e}^{-\gamma(s)}}{\Psi(s)}$ and $g(s)$, we have

$$
g\left(\|u\|_{\infty}\right) \mathrm{e}^{-\gamma(u)} \geq f(x, u) \mathrm{e}^{-\gamma(u)} \geq \frac{f\left(x,\|u\|_{\infty}\right) \mathrm{e}^{-\gamma\left(\|u\|_{\infty}\right)}}{\Psi\left(\|u\|_{\infty}\right)} \Psi(u), \quad \text { a.e. } x \in \Omega
$$

and, since $f\left(x,\|u\|_{\infty}\right)$ is bounded from below and the integrability of $\beta / \alpha$ in a right neighborhood of zero, we derive that

$$
M \geq f(x, u) \mathrm{e}^{-\gamma(u)} \geq-M \Psi(u), \quad \text { a.e. } x \in \Omega
$$

for some positive constant $M$ depending on $\|u\|_{\infty}$. As a consequence, the right hand term of the equations satisfied by $\Psi(u)$ is bounded and so $\Psi(u)$ belongs to $W^{2, p}(\Omega)$ for every $p<\infty$ (see [25]). In particular, since $\Omega$ has the strong local Lipschitz property [1], Paragraph 4.5, by Morrey's inequality (cf. [1], Thm. 5.4, Part II), $\Psi(u) \in C^{1}(\bar{\Omega})$. Taking into account that $\Omega$ satisfies the interior sphere condition in every point of $\partial \Omega$ and using that $-\Delta \Psi(u)+M \Psi(u) \geq 0$ and the Hopf lemma [25], Lemma 3.4, we deduce that $\frac{\partial \Psi(u)}{\partial \nu}<0$ on $\partial \Omega$ and therefore $\frac{\Psi(v)^{2}}{\Psi(u)} \in H_{0}^{1}(\Omega) \cap L^{\infty}(\Omega)$.

Using now identity $(2.5)$ for the solution $u$ with $w=\frac{\Psi(v)^{2}}{\Psi(u)}$ we get

$$
-\int_{\Omega} \alpha(u) \mathrm{e}^{-\gamma(u)}\left(\frac{\Psi(v)}{\Psi(u)}\right)^{2} \Psi^{\prime}(u)|\nabla u|^{2}+2 \int_{\Omega} \alpha(u) \mathrm{e}^{-\gamma(u)} \frac{\Psi(v)}{\Psi(u)} \Psi^{\prime}(v) \nabla u \cdot \nabla v=\int_{\Omega} \frac{\mathrm{e}^{-\gamma(u)} f(x, u)}{\Psi(u)} \Psi(v)^{2},
$$

which can be written as

$$
-\int_{\Omega}\left(\frac{\Psi(v)}{\Psi(u)}\right)^{2}|\nabla \Psi(u)|^{2}+2 \int_{\Omega} \frac{\Psi(v)}{\Psi(u)} \nabla \Psi(u) \cdot \nabla \Psi(v)=\int_{\Omega} \frac{\mathrm{e}^{-\gamma(u)} f(x, u)}{\Psi(u)} \Psi(v)^{2} .
$$

Subtracting (3.1), we deduce that

$$
\int_{\Omega}\left(\frac{\Psi(v)}{\Psi(u)} \nabla \Psi(u)-\nabla \Psi(v)\right)^{2}=-\int_{\Omega}\left(\frac{\mathrm{e}^{-\gamma(u)} f(x, u)}{\Psi(u)}-\frac{\mathrm{e}^{-\gamma(v)} f(x, v)}{\Psi(v)}\right) \Psi(v)^{2},
$$


and so

$$
\int_{\Omega}\left(\frac{\mathrm{e}^{-\gamma(u)} f(x, u)}{\Psi(u)}-\frac{\mathrm{e}^{-\gamma(v)} f(x, v)}{\Psi(v)}\right) \Psi(v)^{2} \leq 0 .
$$

Exchanging the rôles of $u$ and $v$ we get

$$
\int_{\Omega}\left(\frac{\mathrm{e}^{-\gamma(u)} f(x, u)}{\Psi(u)}-\frac{\mathrm{e}^{-\gamma(v)} f(x, v)}{\Psi(v)}\right) \Psi(u)^{2} \geq 0,
$$

and, therefore, subtracting (3.2) from (3.3) we obtain

$$
\int_{\Omega}\left(\frac{\mathrm{e}^{-\gamma(u)} f(x, u)}{\Psi(u)}-\frac{\mathrm{e}^{-\gamma(v)} f(x, v)}{\Psi(v)}\right)\left(\Psi(u)^{2}-\Psi(v)^{2}\right) \geq 0 .
$$

Since the function $\Psi$ is increasing and the function $s \mapsto \frac{f(x, s) \mathrm{e}^{-\gamma(s)}}{\Psi(s)}$ is decreasing, it follows that $\Psi(u)=\Psi(v)$ a.e. in $\Omega$. Using again that the function $\Psi$ is increasing, we conclude the proof.

Remark 3.3. Condition (H3) appearing in the above result turns out to be weaker than assumption (H2) in Theorem 2.9. Indeed, if the function $s \mapsto f(x, s) \mathrm{e}^{-\gamma(s)}$ is decreasing, then the function $s \mapsto \frac{f(x, s) \mathrm{e}^{-\gamma(s)}}{\Psi(s)}$ will also be decreasing. However, while the above proof may only be applied to equations whose principal term is controlled by (almost) linear operators, the argument of Theorem 2.8 is essentially nonlinear and can also be applied to equations of p-Laplacian type.

Acknowledgments. Some of the results in this paper were presented at International Conference on Variational Methods, held in Nankai University, Tianjin, China, from 20 to 26 May of 2007.

The authors wish to thank Prof. J. Carmona for valuable remarks.

\section{REFERENCES}

[1] R.A. Adams, Sobolev spaces. Academic Press, New York (1975).

[2] A. Alvino, L. Boccardo, V. Ferone, L. Orsina and G. Trombetti, Existence results for nonlinear elliptic equations with degenerate coercivity. Ann. Mat. Pura Appl. (4) 182 (2003) 53-79.

[3] D. Arcoya and P.J. Martínez-Aparicio, Quasilinear equations with natural growth. Rev. Mat. Iberoamericana 24 (2008) 597-616.

[4] D. Arcoya, J. Carmona and P.J. Martínez-Aparicio, Elliptic obstacle problems with natural growth on the gradient and singular nonlinear terms. Adv. Nonlinear Stud. 7 (2007) 299-317.

[5] D. Arcoya, J. Carmona, T. Leonori, P.J. Martínez-Aparicio, L. Orsina and F. Petitta, Existence and nonwxistence of solutions for singular quadratic quasilinear equations. J. Differ. Equ. (submitted).

[6] D. Arcoya, S. Barile and P.J. Martínez-Aparicio, Singular quasilinear equations with quadratic growth in the gradient without sign condition. J. Math. Anal. Appl. 350 (2009) 401-408.

[7] G. Barles and F. Murat, Uniqueness and the maximum principle for quasilinear elliptic equations with quadratic growth conditions. Arch. Rational Mech. Anal. 133 (1995) 77-101.

[8] G. Barles and A. Porretta, Uniqueness for unbounded solutions to stationary viscous Hamilton-Jacobi equations. Ann. Scuola Norm. Super. Pisa Cl. Sci. (5) 5 (2006) 107-136.

[9] G. Barles, A.P. Blanc, C. Georgelin and M. Kobylanski, Remarks on the maximum principle for nonlinear elliptic PDEs with quadratic growth conditions. Ann. Scuola Norm. Sup. Pisa Cl. Sci. (4) 28 (1999) 381-404.

[10] P. Bénilan, L. Boccardo, T. Gallouët, R. Gariepy, M. Pierre, J.L. Vázquez, An $L^{1}$-theory of existence and uniqueness of solutions of nonlinear elliptic equations. Ann. Scuola Norm Sup. Pisa Cl. Sci. (4) 22 (1995) 241-273.

[11] M.F. Betta, A. Mercaldo, F. Murat and M.M. Porzio, Existence and uniqueness results for nonlinear elliptic problems with a lower order term and measure datum. C. R. Math. Acad. Sci. Paris 334 (2002) 757-762.

[12] M.F. Betta, A. Mercaldo, F. Murat and M.M. Porzio, Uniqueness of renormalized solutions to nonlinear elliptic equations with a lower order term and right hand side in $L^{1}(\Omega)$. ESAIM: COCV 8 (2002) 239-272

[13] M.F. Betta, A. Mercaldo, F. Murat and M.M. Porzio, Uniqueness results for nonlinear elliptic equations with a lower order term. Nonlinear Anal. 63 (2005) 153-170. 
[14] D. Blanchard, F. Désir and O. Guibé, Quasi-linear degenerate elliptic problems with $L^{1}$ data. Nonlinear Anal. 60 (2005) $557-587$.

[15] L. Boccardo, Dirichlet problems with singular and gradient quadratic lower order terms. ESAIM: COCV 14 (2008) 411-426.

[16] L. Boccardo and L. Orsina, Existence and regularity of minima for integral functionals noncoercive in the energy space. Ann. Scuola Norm. Sup. Pisa Cl. Sci. (4) 25 (1997) 95-130.

[17] L. Boccardo, F. Murat and J.P. Puel, Existence de solutions non bornées pour certaines équations quasi-linéaires. Portugal. Math. 41 (1982) 507-534.

[18] L. Boccardo, F. Murat and J.P. Puel, Résultats d'existence pour certains problèmes elliptiques quasilinéaires. Ann. Scuola Norm. Sup. Pisa Cl. Sci. (4) 11 (1984) 213-235.

[19] L. Boccardo, A. Dall'Aglio and L. Orsina, Existence and regularity results for some elliptic equations with degenerate coercivity. Atti Sem. Mat. Fis. Univ. Modena 46 Suppl. (1998) 51-81.

[20] L. Boccardo, S. Segura de León and C. Trombetti, Bounded and unbounded solutions for a class of quasi-linear elliptic problems with a quadratic gradient term. J. Math. Pures Appl. 80 (2001) 919-940.

[21] H. Brezis and L. Oswald, Remarks on sublinear elliptic equations. Nonlinear Anal. T.M.A. 10 (1986) 55-64.

[22] J. Casado-Díaz, F. Murat and A. Porretta, Uniqueness of the Neumann condition and comparison results for Dirichlet pseudomonotone problems, in The first 60 years of nonlinear analysis of Jean Mawhin, World Sci. Publ., River Edge, NJ (2004) $27-40$.

[23] G. Dal Maso, F. Murat, L. Orsina and A. Prignet, Renormalized solutions of elliptic equations with general measure data. Ann. Scuola Norm. Sup. Pisa Cl. Sci. (4) 28 (1999) 741-808.

[24] D. Giachetti and F. Murat, An elliptic problem with a lower order term having singular behaviour. Boll. Un. Mat. Ital. B (to appear).

[25] D. Gilbarg and N.S. Trudinger, Elliptic Partial Differential Equations of Second Order. Springer-Verlag, New York (1983).

[26] L. Korkut, M. Pašić and D. Žubrinić, Some qualitative properties of solutions of quasilinear elliptic equations and applications. J. Differ. Equ. 170 (2001) 247-280.

[27] A. Porretta, Uniqueness of solutions of some elliptic equations without condition at infinity. C. R. Math. Acad. Sci. Paris 335 (2002) 739-744.

[28] A. Porretta, Some uniqueness results for elliptic equations without condition at infinity. Commun. Contemp. Math. 5 (2003) 705-717.

[29] A. Porretta, Uniqueness of solutions for some nonlinear Dirichlet problems. NoDEA Nonlinear Differ. Equ. Appl. 11 (2004) 407-430.

[30] A. Porretta and S. Segura de León, Nonlinear elliptic equations having a gradient term with natural growth. J. Math. Pures Appl. 85 (2006) 465-492.

[31] S. Segura de León, Existence and uniqueness for $L^{1}$ data of some elliptic equations with natural growth. Adv. Differential Equations 8 (2003) 1377-1408. 